\title{
¿LA POLÍTICA FISCAL DEBE PROMOVERSE DESDE LA POLÍTICA ECONÓMICA COLOMBIANA?
}

\section{FISCAL POLICY MUST BE PROMOTED FROM COLOMBIAN ECONOMIC POLICY?}

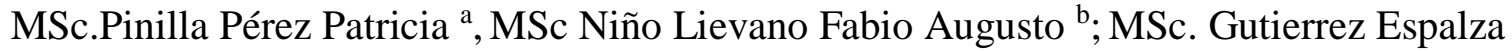 \\ Alejandra Maria ${ }^{\mathrm{c}}$, MBA Suárez Suárez David Andres ${ }^{\mathrm{d}}$
}

${ }^{a}$ Corporación Universitaria Minuto de Dios Uniminuto , grupo de investigación, QUANTUM Calle $28 \mathrm{~N}^{\circ}$ 21-41, Bucaramanga, Colombia, ppinillaper@uniminuto.edu

${ }^{\mathrm{b}}$ Corporación Universitaria Minuto de Dios Uniminuto, Grupo de investigación GIDTI Calle $28 \mathrm{~N}^{\circ}$ 21-41, Bucaramanga, Colombia, fninolievan@uniminuto.edu

${ }^{\mathrm{c}}$ Corporación Universitaria Minuto de Dios Uniminuto, grupo de Investigación GIDTI Calle 28 N$^{\circ}$ 21-41, Bucaramanga, Colombia, Alejandra.gutierrez@uniminuto.edu

d Corporación Universitaria Minuto de Dios Uniminuto , grupo de investigación, QUANTUM Calle 28 N²1-41, Bucaramanga, Colombia, David.suarez@uniminuto.edu

\section{Resumen:}

El resultado de aplicar la política fiscal en la política económica en Colombia, señala que las reformas tributarias, en principio no son enunciadas en las propuestas de los electos candidatos presidenciales, pero en la medida en que se pueda ejecutar el Plan Nacional de Desarrollo, se hace necesario recurrir a buscar nuevos ingresos y esos se aseguran efectivamente, más rápido a través de las reformas tributarias, aplicadas como medidas de política fiscal. 
Los efectos iniciales de la reforma tributaria de Dic 2016, comparado con los beneficios tributarios a empresas que apoyen a ciencia y tecnología y no, para las que generen nuevos empleos y crecimiento en la economía, sobre todo aquellas, de sectores de la economía colombiana, más afectados por la violencia, el conflicto armado y los fenómenos climáticos, con el objetivo de estimular el crecimiento económico y sus efectos se notarían pronto en sector productivo, extractivo y de transformación

Se hace evidente que la política fiscal debe generarse desde los postulados de la política económica, que se debaten en los temas a tratar en el plan de desarrollo propuesto por el presidente de turno de Colombia. Así se cumplirán los indicadores de gestión del presidente de la república en materia de desarrollo económico.

Palabras clave: Elusión, Evasión, Política económica, Política fiscal, Reforma tributaria.

\begin{abstract}
Result of applying fiscal policy to the economic policy in Colombia, points out that tax reforms, in principle, are not stated in the proposals of the elected presidential candidates, but to the extent that the National Development Plan can be implemented, it is necessary to resort to seeking new income and these are effectively ensured, faster through tax reforms, applied as fiscal policy measures.

The initial effects of the tax reform at IDec 2016, compared to tax benefits for companies that support science and technology and not for those generating new jobs and growth in the economy, especially those in sectors of the Colombian economy, more affected by violence, armed conflict and climatic phenomena, with the aim of stimulating economic growth and its effects would soon be noticed in productive, extractive and transformation sector

It is evident that fiscal policy must be generated from the postulates of economic policy, which are discussed in the topics to be discussed in the development plan proposed by the president of shift of Colombia. This will meet the management indicators of the president of the republic in the field of economic development
\end{abstract}

Keywords: Elusion, Evasion, Economic Policy, Fiscal Policy, Tax Reform. 


\section{INTRODUCCIÓN}

El presente artículo de reflexión analiza el efecto del resultado de aplicar la política fiscal en la política económica en Colombia, evidenciando que las reformas tributarias, en principio, no son tenidos en cuenta en las propuestas de candidatos presidenciales, si afectan en la mediada de ejecutar el Plan Nacional de Desarrollo,

Se discute la última reforma tributaria según Ley 1819 del 29 de diciembre de 2016, revisando los conceptos que benefician a las empresas que desarrollen ciencia y tecnología, pero no en la misma media para otro tipo de sector.

Para aclarar el contexto se definen de manera clara los conceptos de política fiscal, política económica, evasión y elusión de impuestos, tópicos importantes que tiene establecidos en una reforma tributaria, como las sanciones penales por incurrir en delitos fiscales.

La política económica se refiere a las acciones que los gobiernos adoptan en el ámbito económico, entre algunas, los sistemas de fijación de tasas de interés y presupuesto del gobierno, el mercado de trabajo, la propiedad nacional, entre otras. Mientras que la política fiscal incluye medidas implementadas por el Gobierno para cumplir sus metas y las del Plan de Desarrollo del presidente a cargo.

El gobierno maneja el volumen y destino del gasto público, por ejemplo, si la economía está teniendo una recesión, puede disminuir los impuestos y aumentar el gasto, si el desempleo tiene una alta tasa, genera nuevos empleos o coloca subsidios de desempleo; si la tasa de inflación es alta, puede disminuir las subida de precios, reduciendo el gatos para contrarrestar la demanda del mercado.

En la política económica, se modifican los impuestos con el fin de modificar la demanda agregada, para estimular el crecimiento económico del país, incluyendo a la población en un economía dinámica, con un legítimo acceso a servicios públicos, educación, capacitación, salud, infraestructura vial, subsidios al desempleo, apoyo a los trabajadores a recuperarse más fácilmente de una pérdida del empleo o de una enfermedad (DINERO, 2017), en otros aspectos a los que tienen derechos los ciudadanos colombianos. Ese debería el ser el objetivo principal de un Plan de Desarrollo Nacional.

¿Cuáles son las razones para realizar una reforma tributaria? La reciente Reforma Tributaria Estructural, (DIAN, 2016) aplicada por la Ley 1819 del 29 de 
diciembre de 2016, fortalece los

mecanismos para la lucha contra la evasión

y la elusión fiscal.

Alguna razones por las que son importantes las reformas tributarias en Colombia, aunque golpee el bolsillo de los más desprotegidos económicamente-, se destaca que Colombia requiere recursos para cubrir las necesidades fiscales, (al 2016 se acercaban a 34 billones de pesos), mejorar el déficit causado por la caída de los precios del petróleo, abonar pagos de intereses de la deuda y disminuir la devaluación del peso colombiano, entre otros.

Otra razón, a destacar, es el control a la evasión de impuestos, que golpea de manera importante las arcas fiscales, según artículo 338 de la Ley 1819 de 2016 adiciona el capítulo 12 del título XV del Código Penal, el cual se ocupa de la omisión de activos o inclusión de pasivos inexistentes, y sanciona con cárcel entre 4 y 9 años a quienes realicen maniobras para reducir su impuesto, además de cuantiosas multas. Según el ministro de Hacienda, Mauricio Cárdenas, se debe combatir la evasión del pago de los impuestos, que en Dic 2016, osciló entre 3\% y 4\% del Producto Interno Bruto -PIB-. (ACTUALICESE. Evasión de impuestos es penalizada con la reforma tributaria, 2017) Colombia es el único país perteneciente a la
Alianza Pacífico que aún no penalizaba dicha práctica ilícita.

A través del tiempo, la historia electoral en Colombia nos demuestra que al iniciar un nuevo periodo presidencial, se diseña el Plan Nacional De Desarrollo, documento en donde se plasma la gestión de desarrollo económico, social y ambiental, es allí, en donde se encuentra la ruta en donde se puede ver el camino para el progreso económico, social, político y cultural de los ciudadanos, cuyo objetivo es mejorar la calidad de vida de la población y garantizar un crecimiento económico de la economía colombiana.

Lo ideal, es que lo propuesto en el Plan Nacional De Desarrollo, para el presente periodo presidencial de Juan Manuel Santos, 2014-2018 "Todos por un nuevo país", sea sostenible en el tiempo, en donde los ciudadanos dejen de utilizar el modelo asistencialista $\quad \mathrm{y}$ promuevan su autosuficiencia, por un lado, obtener acceso a un empleo y un salario digno, en donde puedan satisfacer sus propias necesidades sin depender del gobierno, por otro; lo anterior, hará que la economía colombiana se vuelva más atractiva, generando crecimiento y desarrollo económico, atractivos para la inversión nacional y extranjera. 
La idea central es, que debe haber planificación fiscal del estado a través de las reformas tributarias, con el objetivo de promover la formalización de empresas, ya que eso, generaría nuevos empleos, mayores ingresos y sería sostenible la política fiscal ante el gasto público, y se podría disminuir la deuda externa, la tasa de desempleo e inflación y prevenir crisis y déficits en los sectores productivos y del Estado. Un efecto positivo es el de deducir impuestos a empresas que creen nuevos empleos y abran sucursales o plantas, lo anterior, -aunque parezca un poco desolador que se les descuento impuestos a los grandes empresarios-,conviene porque generarán más empleo, baja el desempleo y la inflación, hay mayor poder adquisitivo, lo que hace aumentar el consumo y el ahorro, y en general se estimula el flujo circular de la economía con la intención de halla un crecimiento generalizado, lo interesante es el control que debe tener el estado con este tipo de empresas, pues seguirán haciendo evasión y elusión de sus impuestos para no generar más ventas ni ganancias para tributar, si no se logra bajar la tarifa de impuestos a las empresas, se afecta la inversión.

Es pertinente aclarar, que la reforma tributaria en la Ley 1819 de 2016 establece disposiciones para la asignación de beneficios tributarios en ciencia, tecnología e innovación -CTI-, a empresas que registren proyectos en Colciencias que desarrollen tecnologías, generen nuevos productos y servicios, incrementen la productividad empresarial e implementen nuevos modelos organizacionales, tecnología e innovación, reforestación y control del medioambiente, inversiones en fuentes de energía no convencional y donaciones; la ley hace referencia a beneficios tributarios a éste tipo de empresas, pero lo ideal sería que apoyen en general a todos los sectores de la economía , sobre todo los más afectados por la violencia, el conflicto armado y los fenómenos climáticos, para estimular el crecimiento económico y sus efectos se notarían pronto en sector productivo, extractivo y de transformación

En la reforma, además, aclara que muchos de los sectores de la economía tienen beneficios específicos y, en general, se les rebaja por generar empleo formal, pero esos beneficios a empresas van desde ingresos no constitutivos de renta, deducciones, rentas exentas y descuentos, según el Director de la Dian, Santiago Rojas, en su artículo_" Gabelas a empresas que la reforma tributaria no quitó"

(ELTIEMPO, 2016) 
Por lo anterior, no hubo una política fiscal, adecuada con la reforma tributaria del 2016, estuvo poco atractiva por el común de la población, al incluir más productos en un impuesto al valor agregado (IVA), o subir el impuesto a ciertos artículos, desestimulando el consumo, la inversión y la producción, lo que encadena a generar menos ingresos, pérdidas económicas, cierre de empresas, desempleo e inflación. La reforma se planteó al contrario del ciclo económico, debió diseñarse primero, los beneficios como la exención o reducción de impuestos a las empresas que generen empleos en ciertos sectores y poblaciones y luego subir los impuestos al consumo

Con la incertidumbre generada por una reforma tributaria, no se ve claro el nivel de confianza de la inversión extranjera en el país, esto golpearía el crecimiento esperado en la economía, en Colombia existe un flujo de inversión extranjera que le permite financiar con comodidad su déficit externo, según (Cárdenas \& Dar, 2016), si no se lograr bajar la tarifa de impuestos a las empresas, se afectaría la inversión:

"las perspectivas sobre la política monetaria de los bancos centrales más grandes del mundo sugieren que sus posturas monetarias permanecerán laxas en 2016 y 2017, por la debilidad del crecimiento de las economías avanzadas más grandes y las preocupaciones por su estabilidad financiera".

\section{CONCLUSIONES}

En definitiva, es necesaria la aplicación de una reforma tributaria planificada y que no atente a los estratos más pobres de la economía, incentivando a promover la formalización de las empresas con el fin de crear nuevos empleos y mayores ingresos para la nación, a través de los recaudos de impuestos, es decir que la política fiscal sea promovida desde una política económica que beneficie el desarrollo económico del país.

SI el motor del desarrollo en Colombia es el sector primario y secundario (Agrario y de trasformación) se debe planear una adecuada reforma tributaria, con deducción o reducción de impuestos a los empresarios que generen nuevos empleos y que apoyen al sector primario de la economía, se tendrán más oportunidades de crecimiento económico, desestimulando , además la informalidad que está acabando con las base pensional de los colombianos y otros aportes parafiscales que se está dejando de percibir el Estado..

\section{BIBLIOGRAFÍA}

ACTUALICESE. Evasión de impuestos es penalizada con la reforma tributaria. ( $2 \mathrm{de}$ 
Enero de 2017). http://actualicese.com.

Obtenido de

http://actualicese.com/actualidad/2017/01/

02/evasion-de-impuestos-es-penalizada-

con-la-reforma-tributaria/

4. Cárdenas, M., \& Dar, J. (21 de 07 de

2016). http://www.dinero.com.

Recuperado el 08 de 11 de 2016, de

http://www.dinero.com/edicion-

impresa/pais/articulo/el-problema-de-la-

inflacion-y-la-reforma-tributaria-en-

colombia/225928

5. DIAN. (29 de Dic de 2016). Ley 1819

Dic 2016. Obtenido de

http://www.dian.gov.co/descargas/centrale

s/2017/Ley_1819.pdf

6. DINERO. (15 de 06 de 2017). Lo que

oide el FMI. Obtenido de

http://www.dinero.com/internacional/artic

ulo/recomendaciones-del-fondo-

monetario-internacional-en-2017/246552

7. ELTIEMPO. (Oct de 2016).

http://www.eltiempo.com. Obtenido de

http://www.eltiempo.com/economia/empre

sas/beneficios-a-empresas-que-mantiene-

la-reforma-tributaria-del-2016-49205

8. http://definicion.de/asistencialismo/\#ixz z4PkiArUUn. (s.f.). Recuperado el 08 de 11 de 2016
9. http://www.banrepcultural.org/blaavirtu al/ayudadetareas/economia/politica_econo mica. (8 de 11 de 2016).

10. http://www.banrepcultural.org/blaavirt ual/ayudadetareas/economia/politica_fiscal . (08 de 11 de 2016).

Osorio-Copete, L. (2016). Reforma tributaria e informalidad laboral en Colombia. Revista Ensayos sobre Política Económica 34, 126-145. R 